\title{
Biophysical characterization of gap-junction channels in HeLa cells
}

\author{
Reiner Eckert $^{1}$, Antonina Dunina-Barkovskaya ${ }^{2}$, Dieter F. Hülser ${ }^{1}$ \\ ${ }^{1}$ Abteilung Biophysik, Biologisches Institut, Universität Stuttgart, Pfaffenwaldring 57, D-70550 Stuttgart, Germany \\ ${ }^{2}$ A. N. Belozersky Institute, Moscow State University, Moscow 119899, Russia
}

Received December 8, 1992/Received after revision and accepted April 6, 1993

\begin{abstract}
HeLa cells seem not to be junctionally coupled when probed with techniques such as Lucifer yellow spreading and/or ionic coupling measured with three inserted microelectrodes. When investigated with double whole-cell patch-clamp measurements, HeLa cells in monolayer cultures were electrically coupled in $39 \%$ of the cases with very low transjunctional conductances (average one to five open channels). These gapjunction channels had a single-channel conductance $\gamma=$ $26 \pm 6 \mathrm{pS}$ and were voltage-gated with an equivalent gating charge $z=3.1 \pm 1.5$ for a voltage of half-maximal inactivation $U_{0}=49 \pm 10 \mathrm{mV}$. The voltage-dependent component represents only $31 \pm 8 \%$ of the total junctional conductance. The voltage-insensitive conductance is characterized by a residual open probability $p_{\mathrm{o}}(\infty)=0.34 \pm 0.12$, which corresponds to a ratio $G_{\text {min }} l$ $G_{\max }=0.50 \pm 0.12$. Dissociation of monolayer cells into cell pairs yielded about $58 \%$ coupled cell pairs with no notably altered single-channel properties.
\end{abstract}

Key words: Intercellular communication - Gap junctions - Connexins - Double whole-cell recording Voltage-dependent gating - HeLa cells

\section{Introduction}

Gap-junction channels bridge the extracellular gap between adjacent eucaryotic cells and connect their intracellular compartments both electrically and metabolically. Two hemichannels - the connexons - are linked by head-to-head alignment and form a communicating gap-junction channel. A connexon is a hexamer of protein subunits which are members of the connexin family. Since connexin $32(\mathrm{Cx} 32)$ was the first gap-junction channel protein to be sequenced [19], it serves as a reference to which all other gap-junction proteins are compared. This connexin family is quite numerous and

Correspondence to: D. F. Hülser more members are likely to be identified. The individual channel conductance varies between $20 \mathrm{pS}$ and $190 \mathrm{pS}$ [12] and gap-junction channels of various tissues differ by their voltage sensitivity (e. g. liver and heart) [21, 25]. The question whether these differences in electrical properties may be attributed to the different connexins expressed in these tissues is now intensively studied (e. g. $[8,15,18,22,28]$ ). A correlation of molecular structure and physiological function is difficult to verify since most cells may express more than one connexin type [5, 26, 27], specific activators or blockers are not available, and functional blocking of gap-junction channels of one connexin type by antibody perfusion faces too many unspecific reactions [18]. In addition, it is still unclear whether a connexon is composed of one connexin type only (homomeric) or whether several connexin types can be assembled to one (heteromeric) connexon. Furthermore, it cannot be decided whether a gapjunction channel consists of two identical connexons (homotypic channel) or of two different connexons (heterotypic channel). These problems may be solved by transfecting cells lacking any expression of connexins with only one connexin type and determining the biophysical characteristics of the resulting gap-junction channels by double whole-cell patch-clamp measurements $[7,8,15,16,25,28]$. In search of cell lines with appropriate properties, we reinvestigated cells that were considered to be not or only to a minor degree junctionally coupled when probed with traditional techniques such as Lucifer yellow spreading and/or ionic coupling measured with three inserted microelectrodes. In our hands, the cell lines SK-HEP-1 [7], L [6], HeLa [10], and $\mathrm{RE}_{\mathrm{e}}[10]$ revealed more or less junctional coupling when probed with the high-resolution patch-clamp method but most of the cells did not tolerate these measurements for a sufficiently long time span.

We report here our experiments with HeLa cells, which best met the required standards: no or low total junctional conductance and high stability under double whole-cell patch-clamp measurements. Both monolayer cultures and isolated pairs of HeLa cells were found to 
be electrically coupled in $39 \%-58 \%$ of the measurements, though with very low transjunctional conductances. HeLa cells tolerated even long-lasting double whole-cell measurements. Together with their low background of gap-junctional conductance and low-single channel conductance this makes them a favourite target cell for transfection with defined connexin genes and subsequent electrophysiological characterization.

\section{Materials and methods}

Cell culture. HeLa (ATCC CCL2) is a permanently growing epithelial-like cell line derived from a human cervix carcinoma. HeLa cells have been maintained in our laboratory since 1970 (see [10]). Cells were cultivated in Dulbecco's modified Eagle's medium (DMEM) supplemented with $3.7 \mathrm{~g} / \mathrm{l} \mathrm{NaHCO}_{3}, 100 \mathrm{mg} / \mathrm{l}$ streptomycin sulphate, $150 \mathrm{mg} / \mathrm{l}$ penicillin $\mathrm{G}$, and $10 \%$ newborn calf serum at $\mathrm{pH} 7.4$ and $37^{\circ} \mathrm{C}$ in a humidified incubator with an $8 \%$ $\mathrm{CO}_{2}$ /air mixture. The cells were grown as monolayers to $70 \%$ confluency in $60-\mathrm{mm}$ plastic petri dishes or on glass cover-slips. During dye-coupling experiments, the cells were kept in phosphatebuffered saline (PBS), and for ionic-coupling measurements they remained in DMEM, corresponding to the standard protocol that is often used to demonstrate junctional coupling [3].

For the preparation of cell pairs, the culture medium was decanted and the cells were rinsed with PBS to remove serum components. After addition of $1 \mathrm{ml}$ ice-cold trypsin $(0.25 \%$ in PBS, calcium- and magnesium-free) to the dish, the cells were incubated for $5 \mathrm{~min}$ at $37^{\circ} \mathrm{C}$. Trypsinization was stopped by adding $2 \mathrm{ml}$ culture medium containing $10 \%$ serum and the cells were dissociated by gentle agitation with a pasteur pipette. Cells were allowed to settle on glass cover-slips (14 mm diameter). After 30 min at $37^{\circ} \mathrm{C}$, when about $80 \%$ of the cells had attached to the glass, a cover-slip was removed from the culture dish, rinsed with PBS, and transferred into an experimental chamber containing PBS or Krebs Ringer solution.

Dye and ionic coupling. Glass micropipettes were pulled from capillary glass (Hilgenberg Glas, Malsfeld, Germany) with a vertical pipette puller (700 C, David Kopf Instruments, Tujunga, USA), and back-filled with a $4 \%(w / v)$ solution of Lucifer yellow $\mathrm{CH}$ (Sigma, St. Louis, USA) in $1 \mathrm{~mol} / \mathrm{l} \mathrm{LiCl}$. The dye was injected iontophoretically into a monolayer cell for $20 \mathrm{~s}$ with a negative current of about $20 \mathrm{nA}$ supplied by the iontophoresis unit of a microelectrode amplifier (L/M-1, modification $500 \mathrm{M} \Omega$, List-Electronic, Darmstadt, Germany). The electrode was withdrawn from the cells and photographs were taken 2 min and $10 \mathrm{~min}$ after the iontophoretic injection was finished.

KCl-filled glass capillaries were used to measure ionic coupling between monolayer cells with three intracellularly inserted electrodes $[10,11]$. These measurements were performed under phase-contrast microscopes and the microelectrodes were operated by micromanipulators with electrical drives (DC3 + STM3, Gebr. Märzhäuser, Wetzlar, Germany). Photographs were taken with an Olympus OM2 camera on Kodak T-MAX400 films with phase contrast and under epifluorescence illumination (Standard, Filter sets 05 or 09, Zeiss, Oberkochen, Germany).

Current recording. We have used the double whole-cell patchclamp technique [14] to study current fluctuations through single gap-junction channels in cell pairs and in monolayer cells. For double whole-cell measurements the pipette solution consisted of $119 \mathrm{mM} \mathrm{KCl}, 2.9 \mathrm{mM} \mathrm{MgCl} 2,5 \mathrm{mM}$ EGTA, and $10 \mathrm{mM}$ HEPES adjusted to $\mathrm{pH} 7.4$. Current recordings were made using two List EPC-7 patch-clamp amplifiers (List Electronic, Darmstadt, Germany). Junctional coupling was tested either by square pulses or ramps of voltage. For current-to-voltage analyses, voltage ramps ( $2.5 \mathrm{~s}$ or $25 \mathrm{~s}$ duration, $0-100 \mathrm{mV}$ amplitude $\stackrel{\wedge}{=} 40 \mathrm{mV} / \mathrm{s}$ or $4 \mathrm{mV} /$ s) were applied to one cell while the neighbouring cell was kept at a constant voltage near its resting potential. Current recordings from both cells were low-pass filtered at $250-500 \mathrm{~Hz}$ and stored on video tape via a modified Sony PCM-502EM (Sony, Fellbach, Germany) digital audio processor [2]. Since this system provides only two d.c. inputs it is not possible to record the currents and voltages for two cells concurrently. For voltage-ramp experiments, therefore, we used the audio input of the video recorder to store trigger pulses, which mark the start and end of each ramp. Since the start and end values for each voltage ramp are known, the respective voltages at each time point can easily be reconstructed numerically.

Data processing. Data processing was performed off-line using an IBM-AT 80386 compatible microcomputer equipped with appropriate A/D-converter hardware (DT2821, DataTranslation, Bietigheim-Bissingen, Germany). Programmes for acquisition and analysis of patch-clamp data were developed in our laboratory according to standard techniques for single-channel analysis [4] using the ASYST programming language (Keithley Asyst, München, Germany). For current-to-voltage analysis the data are sampled in sweeps of 1024 points per trace with the pulse from the audio trace serving as a trigger signal. Each data file consists of an ensemble of 10-100 current records, each of which corresponds to one voltage ramp. Channel conductances were determined manually as the slope of the ridges in current/voltage surfaces [23] of these records. We have also estimated the voltage-dependent open probability of the channel within the range of the voltage ramp, using a modified $I / I_{\max }$ technique. Further details about computer analysis procedures are given in the Appendix. The channel-open probability distribution of the voltage-dependent component was fitted with a Boltzmann distribution of the form:

$p_{\circ}(U)=\frac{1}{1+\mathrm{e}^{\frac{z q_{e}}{k T}\left(U-U_{0}\right)}}$

The equivalent gating charge $z$ and the voltage for half-maximal inactivation $U_{0}$ were estimated and are used together with the channel conductance to characterize the respective gap-junction channel species. All numerical values are given as mean \pm standard deviation.

\section{Results}

When junctional conductance is probed with Lucifer yellow, HeLa monolayer cells retain the dye in the injected cell, as can be seen in Fig. 1. No spread of the tracer can be observed even $10 \mathrm{~min}$ after a single 20 -s injection. For many other cell lines [3] this time span is sufficient to demonstrate dye spreading, which is often already observed during the injection (current) pulse. A more sensitive technique to identify the absence of gap-junctional coupling is the determination of ionic coupling with three microelectrodes. Figure 2 gives an example of such an experiment, where the injected current pulse resulted in a depolarizing pulse superimposed to the membrane potential only in the injected but not in an adjacent cell. With inserted glass microelectrodes the input resistance of the cells is of the order of $10 \mathrm{M} \Omega$ [10]; together with the minimum coupling coefficient [11] of 0.01 the predicted resolution of the junctional conductance is about $1 \mathrm{nS}$. With high-resolution patch-clamp current recordings, junctional conductances exceeding this value have been observed only in $4 \%$ of the cases. In $35 \%$ of the measurements $(n=75)$ conductances of up to $1000 \mathrm{pS}$ were observed in HeLa monolayer cells; the rest had no 

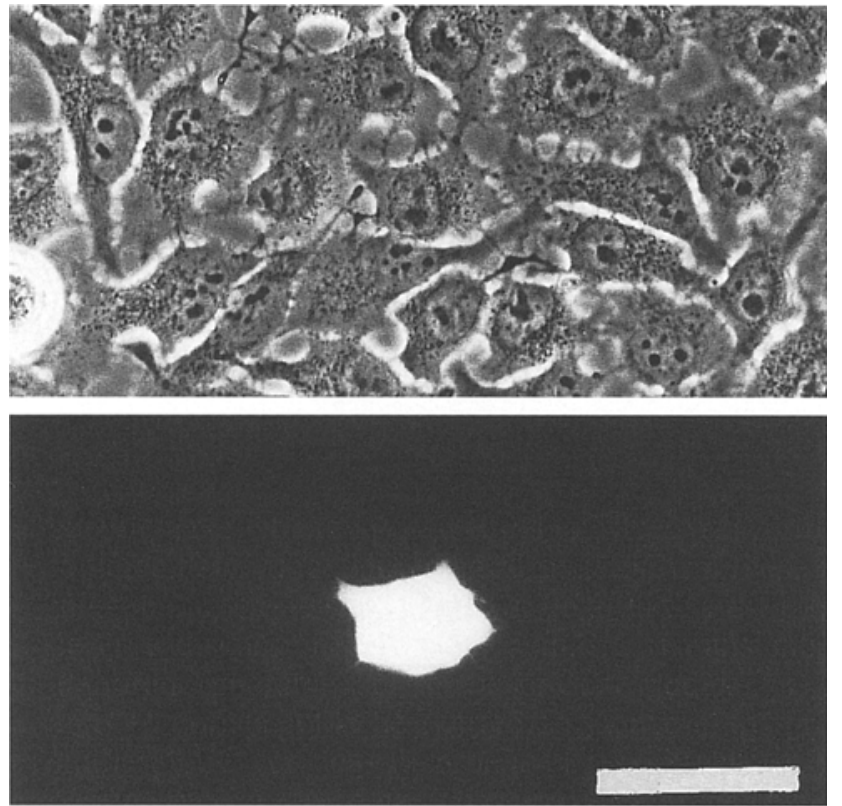

Fig. 1. HeLa monolayer cells probed for gap-junctional communication with Lucifer yellow. Top: phase contrast; bottom: epifluorescence illumination of the same cells. No dye has spread from the injected to neighbouring cells. Horizontal bar: $50 \mu \mathrm{m}$

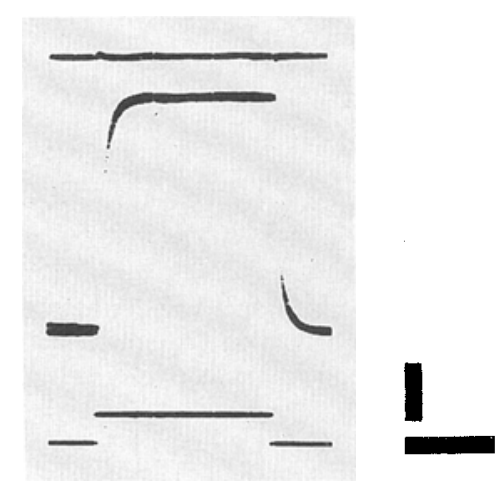

Fig. 2. HeLa monolayer cells probed for gap-junctional communication with three glass microelectrodes. A 5-nA current pulse of $20 \mathrm{~ms}$ duration (lower trace) resulted in a $60-\mathrm{mV}$ depolarization in the injected cell (middle trace) but no deflection was resolved in an attached cell (upper trace). Horizontal bar: $10 \mathrm{~ms}$; vertical bar: $10 \mathrm{nA}$ or $15 \mathrm{mV}$

resolvable coupling. Coupling was observed more often $(58 \%, n=103)$ when junctional conductance was determined in isolated cell pairs (see Fig. 3).

Owing to the low initial conductance, single-channel currents can be observed between neighbouring cells in HeLa monolayers without any pretreatment and in isolated cell pairs. For the record shown in Fig. 4, a constant transjunctional voltage of $50 \mathrm{mV}$ was applied and the channel currents in both cells were recorded. The variance of the background noise is quite large compared to single-channel currents (see also Fig. 5 a), which results in an effective signal-to-noise ratio of about 23 (at $250 \mathrm{~Hz}$ bandwidth). Recordings of single-channel conductances of this size are, therefore, at the resolution limit of our system. Single-channel currents had ampli-

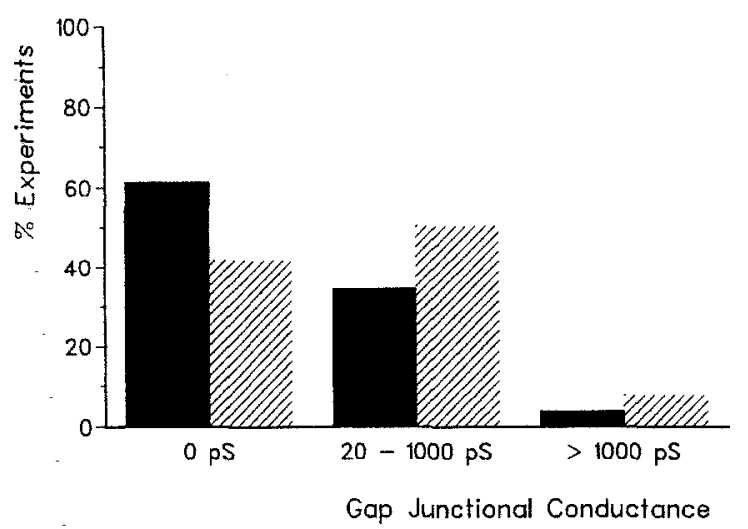

Fig.3. Range of total gap-junctional conductance in monolayers (filled bars, $n=75$ ) and in isolated cell pairs (hatched bars, $n=$ 103) of HeLa cells measured with the double whole-cell patchclamp technique

2 pA
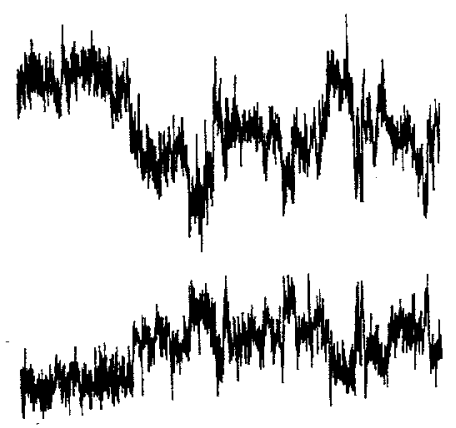

$2.5 \mathrm{~s}$

Fig. 4. Single-channel currents of gap-junction channels between cells of a HeLa cell pair. Upper trace: current record from the cell clamped to a constant value of $50 \mathrm{mV}$. Lower trace: current record in the attached cell clamped to $0 \mathrm{mV}$

tudes of about $1.4 \mathrm{pA}$, corresponding to $28 \mathrm{pS}$ singlechannel conductance, as was determined by an amplitude analysis of a 90-s continuous data recording (Fig. 5). Total conductance was about $110 \mathrm{pS}$ corresponding to about four or five active channels in this cell junction. Measurements at single-channel level reveal that with increasing transjunctional voltages the single-channel conductance is not changed but the open probability is drastically reduced. An amplitude histogram, as shown in Fig. 5 a, can therefore only represent the channels' activity at a given transjunctional voltage. Instead of voltage pulses, ramps can be used which allow a continuous recording of the junctional current over a wide range of voltages. An example for this type of recording is given in Fig. 6, where the transjunctional voltage $U_{\mathrm{j}}$ varied between 0 and $-50 \mathrm{mV}$ according to the difference in the clamped transmembrane potentials $U_{1}$ and $U_{2}$ in cell 1 and cell 2 . The corresponding current $I_{1}$ in cell 1 is the sum of the junctional and non-junctional membrane currents $I_{\mathrm{j}}$ and $I_{\mathrm{m}}$. The current $I_{2}$ in cell 2 corresponds mainly to the junctional current $I_{\mathrm{j}}$, since at $0 \mathrm{mV}$ negligible membrane current is observed.

Gap-junction channel events are mirror-imaged on buth current traces $\left(^{*}\right)$, whereas non-junctional channel 
a

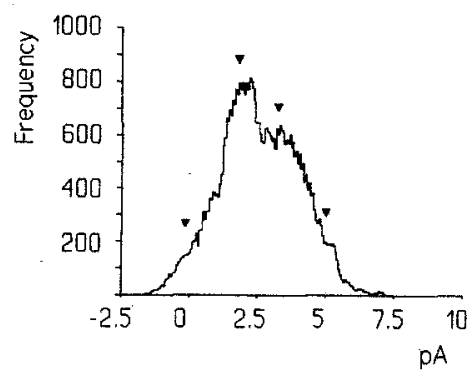

b

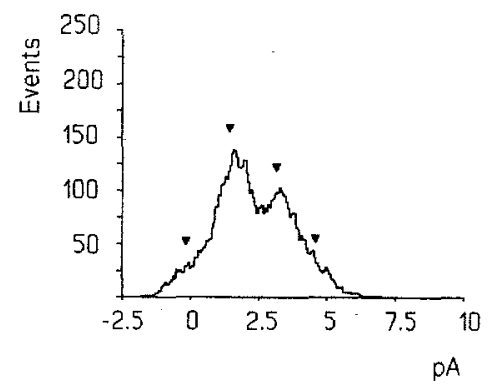

Fig. 5. Amplitude (a) and event histogram (b) of the recording from which Fig. 4 is taken. Histograms contain data from $90 \mathrm{~s}$ continuous sampling at a rate of $500 \mathrm{samples} / \mathrm{s}$. Histograms were calculated using an initial bin width of $0.03 \mathrm{pA} / \mathrm{bin}$ and smoothed by a median filter for display. Peaks can be seen at intervals of about $1.4 \mathrm{pA}$ (arrows) corresponding to a single-channel conductance of $\gamma \approx 28 \mathrm{pS}$

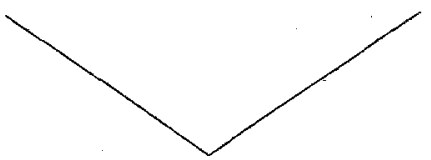

$u_{1}$
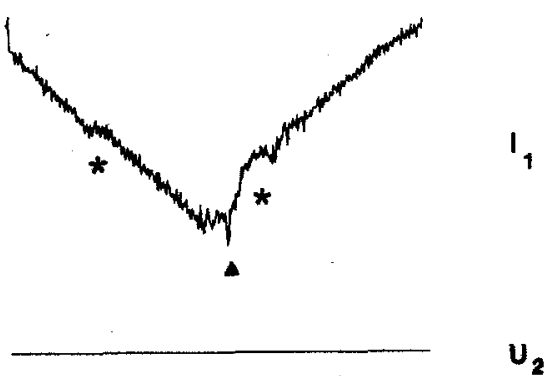

$\mathbf{u}_{2}$

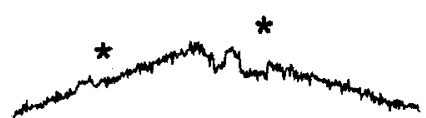

$I_{2}$

Fig. 6. Representative measurement of a voltage ramp experiment used for current/voltage analysis for gap-junctional coupling in HeLa cells. Upper two traces show transmembrane potential $\left(U_{1}\right)$ and whole-cell current $\left(I_{1}\right)$ from cell 1 where voltage ramps of 0 to $-50 \mathrm{mV}$ were applied. Lower two traces are from cell 2 which was clamped to a constant voltage $\left(U_{2}\right)$ of $0 \mathrm{mV}$ near its resting potential. The current recording from this cell $\left(I_{2}\right)$ corresponds mainly to the current flowing through gap-junction channels. Horizontal bar: $1 \mathrm{~s}$; vertical bars: $10 \mathrm{pA}$ or $25 \mathrm{mV}$

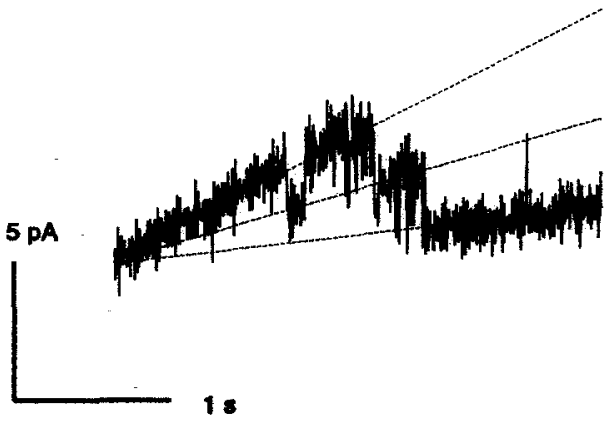

Fig. 7. Single-channel recording from two gap-junction channels in HeLa cells using voltage ramps. A voltage ramp of $2.5 \mathrm{~s}$ duration ranging from 0 to $-100 \mathrm{mV}$ was applied to cell 1 , the resulting currents were recorded from cell 2 clamped to a constant voltage of $0 \mathrm{mV}$

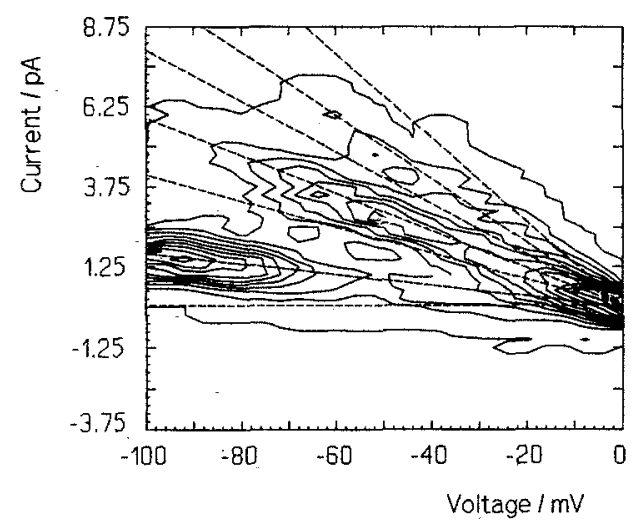

Fig. 8. Current/voltage surface of single gap-junction channel data from HeLa monolayer cells. Current/voltage surface was computed from 20 records of the data set from which Fig. 7 is taken. Fitting straight lines to the ridges of the current/voltage surface was done by eye on a computer screen with coloured contour lines, where the ridges can be better observed than in the small linedrawing presented here. They correspond to six active channels with a mean conductance $\gamma=25 \pm 4 \mathrm{pS}$

events are observed in the respective cell only (e. g. arrow in cell 1). Since only the currents through gap-junction channels follow the pattern of the transjunctional voltage ramp they are best identified in a cell kept at a constant voltage close to the cell's resting potential (Fig. 7). Recordings from these cells are usually less noisy than those from the cells clamped to voltage ramps and, therefore, used for current/voltage analysis.

With some modifications for the calculation of channel-open probabilities, an analysis programme for voltage-dependent gating in ion channels has been developed which also allows the presentation of two-dimensional histograms (current/voltage surfaces, for details see Appendix). We have applied this analysis programme to voltage-dependent currents from single gapjunction channels to estimate both channel conductance and voltage-dependent gating parameters in HeLa cells. With current/voltage surfaces of voltage ramps, singlechannel conductance can be measured more accurately from the slope of the ridges corresponding to the peaks of conventional histograms of stationary data. From the current/voltage surface shown (Fig. 8), single-channel 


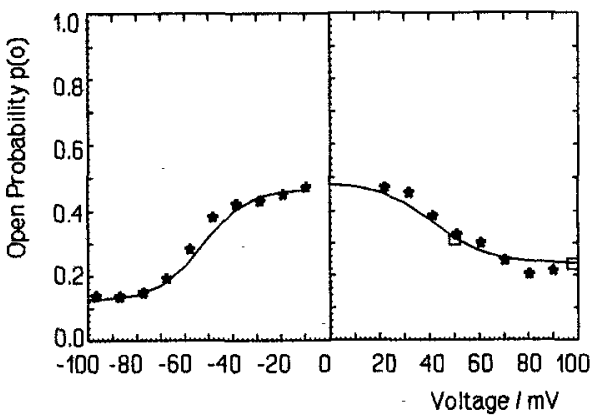

Fig.9. Voltage-dependent open probability of gap-junction channels in HeLa cells. The left side is derived from the data shown in Fig. 8; the right side represents 23 ramps of $2.5 \mathrm{~s}$ duration ranging from 0 to $100 \mathrm{mV}$ from the same experiment. $\square$, Data obtained in voltage-step experiments. —__ A Boltzmann fit to the data assuming two symmetric gates in series. Boltzmann parameters were $z=-2.8$ and $U_{0}=-52.4 \mathrm{mV}$ for the "left" gate, and $z=$ 2.4 and $U_{0}=42.5 \mathrm{mV}$ for the "right" gate

conductances $\gamma=25 \pm 4 \mathrm{pS}$ can be estimated for the gap-junction channels in these HeLa cells. We have pooled the data for single-channel conductance from 16 experiments (stationary data, voltage ramps, and pulses) which resulted in a mean single-channel conductance of $\gamma=26 \pm 6 \mathrm{pS}$.

From the data obtained with voltage-ramp experiments the channel-open probability was estimated for different voltages. Plotting the open probability versus transjunctional voltage revealed voltage-dependent gating in HeLa cells (Fig. 9). The open-probability distribution follows a bell-shaped curve where the maximum open probability is at $0 \mathrm{mV}$ transjunctional voltage, which corresponds to the physiological situation in gapjunctionally coupled cells. This indicates that two voltage-dependent gates (one from each connexon) are in series [24]. The voltage-dependent component is $31 \pm 8 \%$ of the total open probability, so additional voltage-independent component(s) must be present. By fitting only the voltage-dependent component of one gate to a Boltzmann distribution, mean gating parameters for the voltage-dependent gates were evaluated from 8 independent experiments: equivalent gating charge $z=$ $3.1 \pm 1.5$, voltage for half-maximal inactivation $U_{0}=$ $49 \pm 10 \mathrm{mV}$, maximum open probability at $0 \mathrm{mV}$ $p_{\mathrm{o}}(0)=0.66 \pm 0.14$, and residual open probability at $100 \mathrm{mV} p_{\mathrm{o}}(\infty)=0.34 \pm 0.12$, which corresponds to a ratio of $G_{\min } / G_{\max }=0.50 \pm 0.12$. The ramp duration and velocity were chosen according to the time constants of current-relaxation experiments. Using voltage steps of $50 \mathrm{mV}$ and $100 \mathrm{mV}$, time constants of approximately $260 \mathrm{~ms}$ and approximately $31 \mathrm{~ms}$ were estimated respectively, which are small compared to those found in other gap-junction channels [16]. Under these conditions, values for $G_{\mathrm{ss}} / G_{0}$ $G_{\min } / G_{\max } \approx 0.6(50-\mathrm{mV}$ steps) and $0.5(100-\mathrm{mV}$ steps) were determined, which correspond to $p_{\mathrm{o}}(50) \approx 0.3$ and $p_{\mathrm{o}}(100) \approx 0.25$ in Fig. 9 (open squares) and are in reasonable agreement with the ramp data. The observed differences in the Boltzmann parameters between 2.5-s and 25-s ramps had the same variability as was observed in different experiments with
$2.5 \mathrm{~s}$ ramp duration. With the ramp protocol used in our experiments (starting from $U_{\mathrm{j}}=0$ to $U_{\mathrm{j}}=100 \mathrm{mV}$ or $-100 \mathrm{mV}$ ) significant hysteresis between the rising or falling phase of a ramp was observed in some experiments only (data not shown). Hysteresis would indicate non-equilibrium behaviour of the channel as a result of kinetic coupling of the voltage gates, an effect that has been described for amphibian blastomeres [24].

\section{Discussion}

Cells in intact tissues are often coupled by gap junctions; the type of connexin(s), however, varies in different tissues, can be differently expressed after hormonal stimulation [20,29], and may change during development and maturation [5]. It is impossible to measure the conductance of individual gap-junction channels in intact organs or tissues that normally are characterized by a high junctional conductance. This is also true for most cell cultures. The ideal situation for single-channel measurements is given in an isolated cell pair where the electrical paths are simple and - when the cells are small enough - a tolerable background noise from other membrane currents will enable the detection of single gapjunction channel events. Unfortunately, cells that have been cultured for some time may lose their original specific pattern of gap-junction protein(s) and express other connexin(s) not found in the parental tissue [26]. This prevents a correlation between connexin type and biophysical characterization of single gap-junction channel properties since several connexin arrangements in channels may occur.

A better approach to characterize connexin types by their single-channel properties seems to be transfection of non-coupled cells with only one specific connexin type. Since Lucifer yellow has been described as being retainted in the human hepatoma PLC cell line, though there is considerable $(1-2 \mathrm{nS})$ transcellular conductance [17], we reinvestigated several cell lines that had been described as "non-coupled" with the double whole-cell patch-clamp technique. These high-resolution measurements of gap-junctional conductance always revealed some small amount of electrical coupling even in "noncoupled" cell lines. With respect to this low endogenous coupling, our cell lines did not differ from SK-HEP-1 cells, which have already been used in connexin-transfection experiments $[7,8,15,16,25]$. A complete communication deficiency - as was described for N2A cells [28] - could not be observed. We have selected HeLa cells for our further investigations because of their low single-channel conductances, their low endogenous gapjunction conductance, their stability in whole-cell experiments, and because no expression of most of the known connexin genes could be detected (K. Willecke, personal communication), and an appropriate expression vector was available for these cells (K. Willecke, personal communication). For transfection experiments, background coupling should typically be less than about $5 \%$ of the expected coupling rate after transfection, which can easily exceed $20 \mathrm{nS}$. For these cells, the rate 
of incidence of endogenous gap-junction channels should not significantly interfere with single-channel properties of connexins transfected with a high rate of expression.

Single-channel conductances $(\gamma)$ of about $25-30 \mathrm{pS}$ have also been reported in primary heart fibroblasts [22], and the human hepatoma lines PLC [17] and SK-HEP-1 $[15,25]$. So far, single-channel conductance and voltage characteristics $\left(\gamma=26 \mathrm{pS}, U_{0}=49 \mathrm{mV}, z=3.1\right)$ clearly differentiate this channel from those connexins which have been electrophysiologically characterized in transfected SK-HEP-1 [7, 8, 15, 16] and N2A cells [28]. The endogenous cell-cell channel in HeLa cells may well correspond to a not yet identified connexin type, which should also be present in SK-HEP-1 cells [25], however, similar characteristics $\left(\gamma=21-31 \mathrm{pS}, U_{0}=38 \mathrm{mV}, z=\right.$ 2.5) have recently been published for chick Cx45 [28]. Our investigations with transfected HeLa cells will reveal whether the biophysical characteristics for different connexins are identical with those found in different tissues as well as in permanently growing cell lines and will help to analyse the connexin types in these cells.

\section{Appendix}

\section{Analysis of voltage-dependent gating}

Voltage-dependent gating is a common feature found in many ion channels of excitable cells. The biophysical and physiological aspects of this phenomenon have been subject to intensive studies and are well understood both on a structural and on a functional level (sce [9]).

Application of linear voltage ramps is convenient to demonstrate both single-channel conductance and voltage-dependent gating with single-channel records (e. g. [30]), yet no attempt has been made to use this method for the quantification of voltagedependent channel gating from single-channel recordings. Sansom and Mellor [23] introduced two-dimensional amplitude histograms, the so-called current/voltage surfaces, to study voltage-dependent gating of ion channels assembled from two pore-forming peptides in planar lipid bilayers. They suggest the current/voltage surface be fitted with appropriate model equations to estimate voltage-dependent gating parameters. Here we report a different approach, which uses an adaptation of the standard $\hat{I} / I_{\max }$ ratio technique for non-stationary data analysis [1] leading to a more direct estimate of the voltage-dependent open probability.

We have applied this method to analyse voltage-dependent gating in gap-junction channels. However, as it is also applicable for any other voltage-dependent ion channels a general description of this procedure is given in the following.

To give an outline of the theoretical background of this method, let us assume the following simple two-state reaction scheme [9]:

$$
\text { open } \underset{k_{\mathrm{co}}(U)}{\stackrel{k_{\mathrm{oc}}(U)}{\rightleftharpoons}} \text { closed. }
$$

This is a reasonable assumption for gap-junction channels as long as we regard only the voltage-dependent component of one gate. The simplest model that describes gap-junction channel gating requires more kinetic states that are not voltage-dependent. They have, therefore, a constant open probability and are neglected for our analysis of voltage-dependent gating. A complete description of a kinetic model for the gap-junction channel would exceed the scope of this publication.
In Eq. (2) the rates $k_{\mathrm{oc}}$ and $k_{\mathrm{co}}$ are assumed to depend on the apparent voltage $U$ :

$$
\begin{aligned}
& k_{\mathrm{oc}}(U)=\hat{k} \mathrm{e}^{A_{\mathrm{oc}}\left(U-U_{0}\right)} \\
& k_{\mathrm{co}}(U)=\hat{k} \mathrm{e}^{A_{\mathrm{cos}}\left(U-U_{0}\right)} .
\end{aligned}
$$

Where $U_{0}$ is the voltage of half-maximal inactivation when $k_{\mathrm{oc}}=$ $k_{\mathrm{co}}=\hat{k}$ and $A$ gives the voltage sensitivity of the respective rate. The equilibrium constant for this reaction scheme is given as:

$K=\frac{k_{\text {co }}}{k_{\text {oc }}}=\frac{\text { [open] }}{\text { [closed] }}$

which is also equivalent to the ratio of the open and closed probability:

$K=\frac{p_{\mathrm{o}}}{p_{\mathrm{c}}}=\frac{p_{\mathrm{o}}}{1-p_{\mathrm{o}}}$.

From Eq. (4) and (5) the open probability $p_{0}$ of the channel can be calculated as:

$p_{\mathrm{o}}=\frac{\text { [open] }}{\text { [open] }+[\text { closed] }}$

where the square brackets indicate the relative time spent in the respective state.

If the system is in thermodynamic equilibrium, i. e. with stationary recordings, it can be shown, that the probability of open channels follows a binomial distribution with a mean:

$\bar{I}=i N p_{\text {o }}$

where $\bar{I}$ is the average current over a very long (ideally infinite) period, $N$ is the number of active channels and $i$ is the unit current of an open channel. Note that in this case the channel-open probability $p_{\mathrm{o}}$ is constant.

Since the unit current of the channel is $i=\gamma U$ (where $\gamma$ is the unit conductance of the channel), Eq. (7) may be rewritten as:

$\bar{I}=N \gamma U p_{\text {o }}$.

With stationary records, $p_{\mathrm{o}}$ is assumed to be time-invariant. However, Eq. (7) also holds for non-stationary data. Thus, if channel gating is voltage-dependent and if the command voltage is changed, e. g. by applying ramps, Eq. (8) must be written as:

$\hat{I}(U)=N \gamma U p_{\mathrm{o}}(U)$.

Here $\hat{I}$ now represents the average current from an ensemble of similar data [1], which - in the case of voltage-dependent channels - is a non-linear function of voltage. If all channels are open (i. e. $p_{\mathrm{o}}=1$ ), the maximum current is given by

$I_{\max }=N \gamma U$.

Therefore, the open probability can be expressed as the ratio of currents

$p_{\mathrm{o}}(U)=\frac{\hat{I}(U)}{I_{\max }}$

which is equivalent to the ratio $I_{o} / I_{0}$, used in current-relaxation experiments with voltage pulses [1]. With linear voltage ramps, however, $I_{\max }$ and $\hat{I}(U)$ can be obtained for the total voltage range of the ramp within a single experiment where $I_{\max }$ is determined from the current/voltage surface simply as the component with the highest conductance. The values obtained for $p_{\mathrm{o}}$ can then be plotted versus the applied command voltage.

For voltage-gated channels the open-probability distribution can be related to the change in (electrical) energy between open and closed state via the Boltzmann equation

$p_{\circ}(U)=\frac{1}{1+\mathrm{e}^{\frac{z q_{\mathrm{e}}}{k T}\left(U y-U_{0}\right)}}$

where $q_{\mathrm{e}}$ is the elementary charge, $k$ is the Boltzmann constant, and $T$ is the absolute temperature [9]. When $U$ is set to the voltage 
for half-maximal inactivaton, $U_{0}$, the open probability $p_{\mathrm{o}}$ becomes $1 / 2$. The parameter $z$ determines the maximal slope in the decline of the open-probability distribution and is usually interpreted as the valence of an equivalent gating charge that moves across the membrane.

For current-to-voltage analysis the data are sampled in sweeps of 1024 points/trace with the pulse from the audio trace serving as a trigger signal. Each data file consists of an ensemble of 20-100 current records, each of which corresponds to one voltage ramp. To estimate the voltage-dependent open probability of a channel within the range of the voltage ramp, the ensembles are then processed according to the following scheme:

1. Compute the current-to-voltage surface and the average current at each voltage.

2. Determine the baseline and maximum current from the current-to-voltage surface. This is best done manually, since fitting a model function to the surface needs large amounts of computation time.

3. Subtract the baseline from each $I_{\max }$ and $\hat{I}(U)$ at each respective voltage to get the corrected values for these quantities (equivalent to leakage subtraction).

4. Compute the voltage-dependent open probability by dividing $\hat{I}(U)$ by $I_{\max }$.

5. Fit the result with Eq. (12) to estimate $z$ and $U_{0}$. Curve fitting is done using the simplex minimization procedure [13].

For the analysis of gap-junction single-channel currents obtained from the cell clamped to a constant voltage, step 3 may be omitted since under these conditions the baseline is constant (i. e. has zero conductance).

Application of this procedure to data obtained by computer stimulation confirmed that it works reliably, even in situations where conventional methods are difficult to apply. Owing to the finite response time of the channel to voltage changes, some hysteresis between the rising and falling phase of a ramp was expected. Given a $\tau\left(U_{0}\right)$ corresponding to $50 \%$ of the ramp duration, in computer-simulated data the steady-state Boltzmann parameters were reproduced within the $5 \%$ error limit resulting from our fitting procedure (see below). Minor distortions of the steady-state distribution due to hysteresis, therefore, may not be resolved. So far, this method fails to work only for channels with inactivation kinetics or in cases where the apparent time constants exceed the duration of the ramps.

Another problem, which is not inherent to this method, arises from curve fitting of the resulting open-probability distribution with Boltzmann functions. The parameters of the Boltzmann distribution are non-orthogonal, so even for the simple case of a single Boltzmann function, fitting is difficult and the estimated parameters need not be consistent through several repetitions. With the simplex fitting routine used in this study it appears wise to repeat curve fitting several times, with different starting values for the model parameters, to confirm convergence to a global minimum. Though it is possible to some extent to judge convergence from visual inspection of the resulting fit, even in the best cases the fitted parameters were found to deviate by about $5 \%$ from those given for the simulation.

This method was established in our laboratory mainly to facilitate the analysis of voltage-dependent gating of gap-junction channels. Because of the special nature of cell-cell channels, it is not possible to gain access to them with conventional patch-clamp techniques. Instead, a derivative of whole-cell recording is used to measure single-channel currents in isolated cell pairs. In wholecell recording the cell membrane contributes a large amount of the total current, therefore, these records are usually extremely noisy and heavy low-pass filtering has to be applied to increase the signal-to-noise ratio to reasonable values. Filtering reduces the time resolution to levels where conventional kinetic analysis procedures are no longer applicable. It is, therefore, necessary to use steadystate properties to analyse the dynamic behaviour of the channel. In the cell membrane, gap-junction channels are organized in large clusters of more or less tightly packed channels up to several thou- sand per plaque. Multichannel recordings for this channel are, therefore, the rule rather than the exception. Though this situation may also be handled by conventional analysis procedures using steady-state recordings, the new method turned out to be much more efficient and less time consuming in cases where the channel number may be as high as $10-20$ channels per record.

Acknowledgements. We would like to thank Mrs. Beate Rehkopf for expert technical assistance with the cell cultures. This work was supported by a scholarship to A. D.-B. from Boehringer Ingelheim Fonds and to R. E. from Graduiertenförderung des Landes Baden-Württemberg.

\section{References}

1. Aldrich RW, Yellen G (1983) Analysis of nonstationary channel kinetics. In: Sakmann B, Neher E (eds) Single-channel recording. Plenum Press, New York, pp 287-299

2. Bezanilla F (1985) A high capacity data recording device based on a digital audio processor and a yideo cassette re corder. Biophys J 47:437-441

3. Bräuner T, Schmid A, Hülser DF (1990) Tumor cell invasion and gap junctional communication. I. Normal and malignant cells confronted in monolayer cultures. Invasion Metastasis $10: 18-30$

4. Dempster J (1992) Computer analysis of electrophysiological signals. Academic Press, London

5. Dermietzel R, Traub O, Hwang TK, Beyer E, Bennett MVL, Spray DC, Willecke K (1989) Differential expression of three gap junction proteins in developing and mature brain tissues. Proc Natl Acad Sci USA 86:10 148-10152

6. Dertinger H, Hülser DF (1981) Increased radioresistance of cells in cultured multicell spheroids. I. Dependence on cellular interaction. Radiat Environ Biophys 19:101-107

7. Eghbali B, Kessler JA, Spray DC (1990) Expression of gap junction channels in communication-incompetent cells after stable transfection with cDNA encoding connexin 32. Proc Natl Acad Sci USA 87:1328-1331

8. Fishman GI, Moreno AP, Spray DC, Leinwand LA (1991) Functional analysis of human cardiac gap junction channel mutants. Proc Natl Acad Sci USA 88:3525-3529

9. Hille B (1984) Ionic channels of excitable membranes. Sinauer Associates, Sunderland, Mass

10. Hülser DF, Webb DJ (1973) Relation between ionic coupling and morphology of established cells in culture. Exp Cell Res $80: 210-222$

11. Loewenstein WR, Kanno Y (1967) Intercellular communication and tissue growth. I. Cancerous growth. J Cell Biol $33: 225-234$

12. Kolb HA, Somogyi R (1991) Biochemical and biophysical analysis of cell-to-cell channels and regulation of gap-junctional permeability. Rev Physiol Biochem Pharmacol 118:247

13. Nelder JA, Mead R (1965) A simplex method for function minimization. Computer J 7:308-313

14. Neyton J, Trautmann A (1985) Single-channel currents of an intercellular junction. Nature 317:331-335

15. Moreno AP, Eghbali B, Spray DC (1991) Connexin32 gap junction channels in stably transfected cells: unitary conductance. Biophys J 60:1254-1266

16. Moreno AP, Eghbali B, Spray DC (1991) Connexin32 gap junction channels in stably transfected cells: equilibrium and kinetic properties. Biophys J 60:1267-1277

17. Paschke D (1989) Gap Junction Kanäle mit unterschiedlichen Leitfähigkeiten. Dissertation, Universität Stuttgart

18. Paschke D, Eckert R, Hülser DF (1992) High-resolution measurements of gap-junctional conductance during perfusion with anti-connexin antibodies in pairs of cultured mammalian cells. Pflügers Arch 420:87-93 
19. Paul DL (1986) Molecular cloning of cDNA for rat liver gap junction protein. J Cell Biol 103:123-134

20. Risek B, Guthrie S, Kumar N, Gilula NB (1990) Modulation of gap junction transcript and protein expression during pregnancy in the rat. J Cell Biol 110:269-282

21. Rook MB, Jongsma HJ, van Ginneken ACG (1988) Properties of single gap junction channels between isolated neonatal rat heart cells. Am J Physiol 255:H770-H782

22. Rook MB, Jongsma HJ, de Jonge B (1989) Single channel currents of homo- and heterologous gap junctions between cardiac fibroblasts and myocytes. Pflügers Arch 414:9598

23. Sansom MSP, Mellor IR (1990) Analysis of the gating of single ion channels using current-voltage surfaces. J Theor Biol $144: 213-223$

24. Spray DC, White RL, Campos de Carvalho A, Harris AL, Bennet MVL (1984) Gating of gap junction channels. Biophys J $45: 219-230$

25. Spray DC, Moreno AP, Eghbali B, Chanson M, Fishman GI (1992) Gating of gap junction channels as revealed in cells stably transfected with wild type and mutant connexin cDNAs. Biophys J 62:48-50

26. Stutenkemper R, Geisse S, Schwarz HJ, Look J, Traub O Nicholson BJ, Willecke K (1992) The hepatocyte-specific phenotype of murine liver cells correlates with high expression of connexin 32 and connexin 26 but very low expression of connexin43. Exp Cell Res 201:43-54

27. Traub O, Look J, Dermietzel R, Brümmer F, Hülser D, Willecke $\mathrm{K}$ (1989) Comparative characterization of the $21-\mathrm{kD}$ and 26-kD gap junction proteins in murine liver and cultured hepatocytes. J Cell Biol 108:1039-1051

28. Veenstra RD, Wang H-Z, Westphale EM, Beyer EC (1992) Multiple connexins confer distinct regulatory and conductance properties of gap junctions in developing heart. Circ Res $71: 1277-1283$

29. Winterhager E, Stutenkemper R, Traub O, Beyer E, Willecke $\mathrm{K}$ (1991) Expression of different connexin genes in rat uterus during decidualization and at term. J Cell Biol 55:133-142

30. Yellen $\mathrm{G}$ (1983) Single $\mathrm{Ca}^{2+}$-activated nonselective cation channels in neuroblastoma. Nature 296:357-359 